\title{
Validez y reproducibilidad de un cuestionario de actividad e inactividad física para escolares de la ciudad de México
}

\author{
Bernardo Hernández, D.Sc., ${ }^{(1)}$ Steven L. Gortmaker, Ph.D., ${ }^{(2)}$ N an M. Laird, D.Sc., ${ }^{(3)}$ Graham A. Colditz, Ph.D., \\ Socorro Parra-Cabrera, M. en C., ${ }^{(1)}$ Karen E. Peterson, D.Sc. ${ }^{(5)}$
}

\begin{abstract}
Hernández B, Gortmaker SL, Laird NM, Colditz GA, Parra-Cabrera S, Peterson KE. Validez y reproducibilidad de un cuestionario de actividad e inactividad física para escolares de la ciudad de México. Salud Publica Mex 2000;42:315-323.
\end{abstract}

\section{Resumen}

Objetivo. Evaluar la validez y reproducibilidad de un cuestionario autoaplicado de actividad e inactividad física en escolares de 10 a 14 años de la ciudad de México. Material y métodos. Se desarrolló un cuestionario autoaplicado sobre la actividad e inactividad física que se aplicó dos veces a una muestra de 114 estudiantes, de 10 a 14 años de edad, en una población de bajos y medianos ingresos de la ciudad de México, entre mayo y diciembre de 1996. Las madres de los estudiantes llenaron el mismo cuestionario, mientras que aquéllos completaron dos recordatorios de actividad física de 24 horas, que se usaron como criterio de comparación. Se calcularon medidas de tendencia central y de dispersión y se estimó correlación de Pearson. Resultados Las correlaciones entre las horas al día dedicadas a la actividad e inactividad física del cuestionario de los estudiantes y las de los recordatorios de 24 horas ajustadas por edad, sexo, zona de residencia y enfermedad anterior a la administración del cuestionario fueron de 0.03 para la actividad moderada, de 0.15 para la actividad vigorosa y de $0.51(p=0.001)$ para el tiempo dedicado a ver televisión. Al comparar con los recordato rios de 24 horas, el cuestionario sobrestimó el tiempo de ver televisión, leer o participar
Hernández B, Gortmaker SL, Laird N M, Colditz GA, Parra-Cabrera S, Peterson KE. Validity and reproducibility of a physical activity and inactivity questionnaire for Mexico City's schoolchildren.

Salud Publica Mex 2000;42:315-323.

\begin{abstract}
A bstract
Objective. To assess the validity and reproducibility of a self-repo rted questionnaire on physical activity and inactivity, developed for children aged 10-14 in Mexico City. Material and methods. Between May and December 1996, a selfreported physical activity and inactivity questionnaire was developed and applied twice to a sample of 114 students aged 10 to 14, from a low and middle income population of Mexico City. The children's mothers completed the same questionnaire, and two 24-hour recalls of physical activity were used for comparison. Statistical analysis consisted of central tendency and dispersion measures and Pearson's correlation co efficient. Results. Correlations between hours per day spent in physical activity and inactivity from the children's questionnaire and the 24-hour recall data, were 0.03 for moderate activity, 0.15 for vigorous activity, and $0.51(p=0.001)$ for watching television, adjusted by age, gender, town, and illness prior to the administration of the questionnaire. Compared to the 24-hour recall data, the questionnaire overestimated the time spent watching television, reading or participating in vigo ro us activity, and underestimated the time engaged in moderate activity. Statistically significant $(p<0.05)$ six-month reproducibility
\end{abstract}

Este estudio fue financiado por el Consejo N acional de Ciencia y Tecnología de México, la Fundación Mexicana para la Salud, la Fundación W.K. Kellogg y el Instituto N acional de Salud Pública de México.

(1) Dirección de Epidemiología, Instituto N acional de Salud Pública, México.

(2) Department of Health and Social Behavior, Harvard School of Public Health, Boston (MA), Estados Unidos de América (EUA).

(3) Department of Biostatistics, Harvard School of Public Health, Boston (MA), EUA.

(4) Channing Laboratory, Department of Medicine, Brigham and Women's Hospital and Harvard Medical School. And Department of Epidemiology, Harvard School of Public Health, Boston (MA), EUA.

(5) Department of Maternal and Child Health, Harvard School of Public Health, Boston (MA), EUA.

Fecha de recibido: 4 de mayo de 2000 - Fecha de aprobado: 28 de julio de 2000

Solicitud de sobretiros: Dr. Bernardo Hernández Prado. Dirección de Epidemiología, Instituto N acional de Salud Pública. Avenida Universidad 655, colonia Santa María A huacatitlán, 62508 Cuernavaca, Morelos, México.

Correo electrónico: bhernand@ insp3.insp.mx 
en actividades vigorosas, y subestimó el tiempo de actividad moderada. Se observaron coeficientes de reproducibilidad en seis meses aceptables para el tiempo de ver televisión ( $r=0.53)$, dormir $(r=0.40)$, actividad moderada $(r=0.38)$ y actividad vigorosa $(r=0.55)(p<0.05)$. Conclusiones. Entre los estudiantes de 10 a 14 años de la ciudad de México, el cuestionario mostró validez aceptable para estimar el tiempo de ver televisión, y una reproducibilidad aceptable de las medidas del tiempo de ver televisión, de la actividad moderada y de la vigorosa.

Palabras clave: esfuerzo físico; inactividad física; cuestionarios; reproducibilidad de resultados; México values were observed for watching television ( $r=0.53)$, sleeping $(r=0.40)$, moderate $(r=0.38)$, and vigorous activity $(r=0.55)$. Conclusions A mong children of Mexico C ity aged 10-14, the questionnaire showed acceptable validity in estimating the time watching television, and acceptable reproducibility of the time watching television, vigorous and moderate activity.

Key words: exertion; physical inactivity; questionnaires; reproducibility of results; Mexico
$L$ a actividad e inactividad física están asociadas con problemas de salud que incluyen obesidad, enfermedad coronaria, diabetes, cáncer de colon y mortalidad por causas múltiples. ${ }^{1}$ En la transición en la salud que México está experimentando estas enfermedades se han vuelto la principal causa de muerte, y la obesidad ha alcanzado altas tasas de prevalencia . ${ }^{2,3}$ Esto subraya la necesidad de estudiar los factores de riesgo para la obesidad y las enfermedades crónicas. ${ }^{4}$

La actividad e inactividad física son los componentes más variables del gasto energético y están, hasta cierta medida, bajo control voluntario. ${ }^{5}$ Se les ha conceptualizado como áreas diferentes, ${ }^{6}$ ya que pueden afectar la salud mediante diversos mecanismos. La mayoría de los habitantes de países industrializados realiza actividad vigorosa durante muy poco tiempo al día, mientras que pasa mucho tiempo de sedentarismo en el trabajo. ${ }^{7}$ Ver televisión (TV) es una forma de inactividad, y se ha documentado su asociación con la obesidad..$^{8-10}$ La TV puede aumentar el riesgo de obesidad ya sea por una reducción en el gasto de energía o por cambios en la dieta. ${ }^{11}$ En estudiantes mexicanos se han encontrado altos índices de tiempo dedicado a ver TV. ${ }^{10,12}$

Los métodos para medir la actividad física de los estudiantes incluyen sensores de movimiento, ${ }^{13}$ observación, ${ }^{14}$ así como informes individuales o de un sustituto (diarios de actividad física, recordatorios o cuestionarios).${ }^{15}$ El método de agua doblemente marcada ha sido reconocido como válido para evaluar gasto energético al proporcionar un indicador de actividad física; aunque este método es más costoso y de difícil aplicación. ${ }^{16}$ Los cuestionarios de autoinforme acerca de la actividad física son una opción para estudios epidemiológicos con grandes poblaciones; sin embargo, es posible que su validez se vea afectada por problemas de memoria, y que pueda variar según el sexo, la edad y el desarrollo cognitivo de los sujetos. ${ }^{17}$
Hasta donde tenemos conocimiento no se han evaluado la validez y reproducibilidad de cuestionarios para medir la actividad e inactividad física de los estudiantes mexicanos. ${ }^{11}$ El propósito de esta investigación es evaluar la validez y reproducibilidad de un cuestionario autoadministrado sobre actividad e inactividad física desarrollado para estudiantes de 10 a 14 años de la ciudad de México.

\section{Material y métodos}

El estudio se realizó en una población de bajos ingresos (Iztapalapa) y en una de medianos ingresos (Miguel Hidalgo) en la ciudad de México, entre mayo y diciembre de 1996. Al principio del estudio se trabajó con una muestra de 114 estudiantes, de 10 a 14 años de edad, que iban en quinto grado de primaria y primero de secundaria en dos escuelas públicas de la población de bajos ingresos y en dos escuelas privadas de la de medianos ingresos, que fueron elegidas al azar entre 33 escuelas posibles. Para reclutar a los participantes se distribuyó una carta de consentimiento informado para ellos y sus padres. Los procedimientos del estudio fueron aprobados por el Comité de Sujetos Humanos de la Escuela de Salud Pública de Harvard, por el Instituto Nacional de Salud Pública de México y por las autoridades escolares.

Se formaron cuatro grupos focales de 8 a 10 estudiantes de las escuelas participantes, los que proporcionaron información sobre sus actividades físicas usuales. Posteriormente, se adaptó un cuestionario autoadministrado sobre la actividad e inactividad física con base en instrumentos anteriores para hacer referencia a estas actividades. ${ }^{18} \mathrm{El}$ cuestionario se aplicó dos veces a los estudiantes en la escuela, la primera vez entre mayo y junio de 1996, y la segunda, entre noviembre y diciembre de 1996. Personal capacitado ${ }^{19}$ midió talla y peso en la segunda aplicación del cues- 
tionario, siguiendo un protocolo estándar. ${ }^{20}$ También se obtuvieron dos recordatorios de actividad e inactividad física de 24 horas de los estudiantes entre septiembre y octubre de 1996, y se aplicó un cuestionario a las madres en el que se les preguntó sobre la actividad física de sus hijos entre julio y agosto de 1996. Todos los instrumentos de recolección se aplicaron durante las mañanas.

El cuestionario de actividad e inactividad física (Cuestionario de actividad e inactividad de los estudiantes mexicanos, CAINM) fue elaborado siguiendo el formato del Youth Activity Questionnaire (YAQ)* adaptado de un cuestionario de actividad validado en adultos. ${ }^{21,22}$ El cuestionario consta de 14 ítemes que estiman las horas al día dedicadas a realizar actividad moderada (en términos de equivalentes metabólicos -EM-, puntaje de EM/hora entre 3.0 y 5.9) y a actividad vigorosa (puntaje de EM/hora $\bullet 6.0)^{23}$ durante el mes anterior sin distinguir entre días de entre semana y de fin de semana. Una pregunta abierta daba la opción de añadir otra actividad usual.

Se evaluaron las horas de ver TV y otros videos con preguntas adaptadas de un cuestionario sobre tiempo de ver televisión, el Television and Video Measure (TVM). ${ }^{18}$ Se pidió a los estudiantes indicar el tiempo durante el cual veían programas de TV cada día de la semana, y el tiempo que dedicaban a jugar videojuegos, a ver películas en días hábiles y fines de semana durante el mes anterior. Los ítemes fueron ponderados y sumados para obtener una estimación total de tiempo al día viendo TV. Para evaluar el tiempo dedicado a leer o hacer tareas escolares para días entre semana y en fines de semana se usó el mismo formato de preguntas. El tiempo dedicado a dormir se evaluó al preguntar la hora en que los estudiantes iban a dormir y se levantaban en días entre semana y fines de semana. El cuestionario incluía preguntas sobre edad, sexo, grado y cualquier enfermedad que pudo haber interferido con las actividades del estudiante durante el mes anterior.

Se calculó el índice de masa corporal (IMC, peso en kilogramos/talla en metros al cuadrado). Los estudiantes fueron clasificados como con bajo peso, peso normal o sobrepeso usando como puntos de corte los percentiles 15 y 85 específicos de edad y sexo de la distribución de la National Health and Nutrition Examination Survey I (NHANESI). ${ }^{24,25}$ Se obtuvieron los índices de actividad e inactividad multiplicando el costo energético de cada actividad (definida en $\mathrm{EM}^{23}$ ) por la duración de su práctica. Se usaron valores de EM para cada actividad con base en un compendio de Ainsworth y colaboradores. ${ }^{23}$

Los recordatorios de actividad física de 24 horas se obtuvieron mediante entrevistas en las escuelas durante días de clase o en las casas de los estudiantes durante días de asueto (incluyendo sábados, domingos y días festivos). El 98\% de las entrevistas se llevó a cabo de lunes a viernes. Los recordatorios reunían información sobre las actividades realizadas durante días de clases $(84 \%)$ y días de asueto $(16 \%)$. Al seguir el formato de instrumentos anteriormente desarrollados* nutriólogos capacitados preguntaron a los estudiantes sobre sus actividades físicas durante las 24 horas anteriores a la entrevista, con el día dividido en periodos de 30 minutos. Se calcularon los puntajes de EM-horas/día para inactividad, actividad moderada y vigorosa de los datos del recordatorio.

Se aplicó una versión adaptada del CAINM a las madres u otro adulto que viviera con los estudiantes y conociera sus actividades. Este cuestionario tenía formato y contenido idénticos al CAINM, y preguntaba al adulto sobre las actividades del niño. Una entrevistadora llevó el cuestionario a la casa del adulto informante, esperó a que éste lo completara y se lo llevó inmediatamente. A los estudiantes cuyo informante adulto informó valores de actividad/inactividad no plausibles se les identificó como se describe anteriormente $(n=2)$.

El análisis estadístico se llevó a cabo utilizando los paquetes estadísticos Stata versión 4.0 y SAS. Para evaluar la validez de las medidas se estimaron las correlaciones de Pearson y se compararon las medidas de tiempo (horas/día) dedicado a la inactividad, actividad física moderada y actividad física vigorosa, como se informó en las tres fuentes: el segundo CAINM, el informe de las madres sobre la actividad de los estudiantes, y el promedio de los dos recordatorios de 24 horas. En el análisis se incluyeron los valores de un recordatorio para los estudiantes que sólo contestaron uno de los recordatorios $(n=8)$. Los recordatorios fueron el criterio de comparación.

Se realizó un análisis de medidas repetidas usando la rutina SAS Proc Mixed. Esta técnica permite incluir información de todos los sujetos que tuvieron al menos una observación en la variable de resultado de interés y de todas las covariables del estudio, ya que los valores perdidos se estiman de acuerdo con la distribución de las otras variables en el modelo. ${ }^{26-28}$

\footnotetext{
* Field A, Colditz GA, Fox MK, Bosch RJ, Peterson KE. Department of Nutrition, Harvard School of Public Health, Boston (MA), Estados Unidos de América, 1998. Comunicación personal.
} 
Se ajustaron modelos para comparar las medidas de tiempo dedicado a cada forma de actividad o inactividad de acuerdo con las tres fuentes de información y se estimaron las correlaciones entre ellos. Los modelos utilizados incluían el tiempo dedicado a actividad/inactividad como variable dependiente, y la fuente de información, edad, sexo, ubicación de la escuela, enfermedad anterior a la aplicación del cuestionario e IMC como covariables.

Las correlaciones observadas entre las medidas de actividad e inactividad física se ven afectadas por la variabilidad intraindividual, particularmente en los recordatorios de 24 horas..$^{28}$ Por esta razón, se calcularon los índices de variabilidad usando la información de dos recordatorios de actividad física, ${ }^{29}$ y se estimaron las correlaciones deatenuadas ${ }^{30}$ entre los informes de los cuestionarios y los recordatorios de 24 horas.

Para evaluar la reproducibilidad del cuestionario en un periodo de seis meses se realizó el análisis de medidas repetidas para comparar las respuestas de los estudiantes en la primera y segunda aplicación del cuestionario y se estimaron las correlaciones entre sus respuestas ajustando por edad, sexo, ubicación de la escuela, enfermedad anterior a la aplicación del cuestionario e IMC.

El análisis para evaluar la validez y confiabilidad del cuestionario se efectuó excluyendo el caminar como una forma de actividad moderada y se analizó por separado, tomando en cuenta que las dificultades para su informe pueden disminuir su validez.* Se replicó dicho análisis excluyendo a los individuos con valores de actividad e inactividad no plausibles, y usando como variables dependientes los EM-hora/día de actividad. Finalmente, el análisis se realizó estratificando por grado, sexo, ubicación de la escuela y por día hábil o de asueto (sólo para las formas de inactividad) para identificar las diferencias en validez y reproducibilidad a través de los estratos.

\section{Resultados}

El estudio incluyó 114 estudiantes. La tasa de participación fue de $83 \%$, y fue mayor en la población de bajos ingresos (88\%) que en la de medianos ingresos (76\%). Las tasas de respuesta a los diferentes componentes del estudio variaron: 113 estudiantes completaron la primera aplicación del CAINM y 105 la segunda; 98 cuestionarios de las madres fueron llenados ( $88 \%$ por

\footnotetext{
* Field A, Colditz GA, Fox MK, Bosch RJ, Peterson KE. Department of Nutrition, Harvard School of Public Health, Boston (MA), Estados Unidos de América, 1998. Comunicación personal.
}

las madres y $12 \%$ por otro adulto), y 108 estudiantes participaron en al menos un recordatorio de 24 horas (100 estudiantes llenaron dos). Se midió talla y peso de 95 estudiantes.

La edad promedio de los estudiantes fue de $11.7 \pm 1.0$ años, $41 \%$ del sexo masculino y $67 \%$ de la población de bajos recursos. Al principio del estudio, $47 \%$ de los estudiantes de la muestra iban en quinto grado de primaria y $53 \%$ en primero de secundaria. La medida del IMC de los estudiantes fue de $21.3 \pm 3.4$ $\mathrm{kg} / \mathrm{m}^{2}, 5 \%$ fueron considerados de bajo peso, $56 \%$ de peso normal y $39 \%$ con sobrepeso. Quince por ciento de los estudiantes informaron en alguno de los cuestionarios haber padecido una enfermedad que interfirió con sus actividades durante el mes anterior (cuadro I).

Se identificaron nueve estudiantes con valores de actividad e inactividad física no plausibles (suma de estimaciones de horas/día de ver TV, leer y actividad física $\bullet 10.5$ horas/día en días de escuela y 13.5 en días de asueto).

\section{Cuadro I \\ Características de estudiantes de la ciudad DE MÉxico, de 10 a 14 años de edad, QUe Participan en EL estudio. MéXico, 1996}

\begin{tabular}{lcc} 
Variable & No. & $\%$ \\
$\begin{array}{l}\text { Sexo } \\
\text { Masculino }\end{array}$ & 47 & 41 \\
\hline Femenino & 67 & 59 \\
Grado* & & \\
$\quad$ Q uinto (escuela primaria) & 54 & 47 \\
\hline Primero (escuela secundaria) & 60 & 53 \\
Ubicación de la escuela & & \\
$\quad$ Zona de bajos ingresos & & \\
\hline Zona de medianos ingresos & 76 & 67 \\
Estado nutricio ${ }^{\ddagger}$ & 38 & 33 \\
$\quad$ Bajo peso & & \\
\hline Peso normal & 6 & 5 \\
\hline Sobrepeso & 64 & 56 \\
\hline
\end{tabular}

Padeció enfermedad que interfirió

con la actividad antes de aplicar el cuestionario

\begin{tabular}{lll} 
Sí & 17 & 15 \\
\hline No & 97 & 85
\end{tabular}

$\mathrm{n}=114$

* Grado al principio del estudio, mayo de 1996

$\ddagger n=95$ medido al final del estudio, octubre de 1996. Los puntos de corte para el bajo peso, peso normal o sobrepeso corresponden a los percentiles 15 y 85 de distribución de la $\mathrm{N}$ ational Health and $\mathrm{N}$ utrition Examination Survey 124,25 
El tiempo promedio de ver TV, como se estimó en el segundo CAINM, fue de 4.5 horas/día. Los estudiantes pasaron en promedio 2.4 horas/día viendo programas de TV, 0.9 horas/día jugando videojuegos y 1.1 horas/día viendo videos/películas en la videograbadora. Las principales actividades contribuyentes al resultado total de EM-horas/día para la actividad vigorosa, entre los hombres, fueron jugar futbol y correr $\mathrm{y}$, entre las mujeres, correr y jugar basquetbol. Para ambos sexos, los principales contribuyentes a la actividad moderada fueron caminar y hacer quehaceres domésticos.

Se reunió información de 111 estudiantes con datos de actividad e inactividad en al menos una de las fuentes de información (segunda aplicación del CAINM, cuestionario de las madres o recordatorio de 24 horas); 91 estudiantes proporcionaron datos completos en los tres instrumentos. Ya que los resultados del análisis no variaron después de la transformación de las variables o la eliminación de valores de actividad e inactividad física no plausibles, sólo se presentan los resultados de la muestra completa.

Comparados con los recordatorios de 24 horas, tanto los cuestionarios de los estudiantes como los de las madres sobrestimaron el tiempo dedicado a ver
TV, leer, y de práctica de actividad física vigorosa, y subestimaron el tiempo dedicado a actividad física moderada y caminar. Las madres sobrestimaron el tiempo dedicado a dormir, comparado con las estimaciones basadas en los recordatorios de los estudiantes. Todas estas diferencias fueron estadísticamente significativas $(p<0.05)$ (cuadro II). Los estudiantes de la población de bajos recursos sobrestimaron el tiempo dedicado a leer/hacer tareas y a actividad moderada. Los hombres sobrestimaron la actividad vigorosa más que las mujeres.

$\mathrm{Al}$ estimar las correlaciones entre el CAINM y los recordatorios de 24 horas ajustadas para edad, sexo, ingresos y enfermedad anterior a la aplicación del CAINM, sólo se encontraron correlaciones ajustadas estadísticamente significativas para el tiempo de ver TV ( $r$ ajustada $=0.51, r$ deatenuada $=0.69, p<0.05)$. Las correlaciones ajustadas para el tiempo de dormir, leer/ hacer tareas, y actividad moderada o vigorosa no fueron significativas siendo éstas de 0.02 a 0.15 . Las correlaciones ajustadas entre los cuestionarios de estudiantes y madres para medidas de inactividad, actividad moderada y vigorosa fueron de 0.06 para la actividad total excluyendo caminar a 0.39 para el tiempo de dormir. Las correlaciones entre el cuestionario

\section{Cuadro II \\ MEdias CRUdAS Y AJUSTAdAS DE HORAS/Día QUE LOS ESTUdIANTES DEDICAN A ACTIVIDAD FÍSICA E INACTIVIDAD, COMO SE ESTIMÓ EN LA SEGUNDA APLICACIÓN DEL CUESTIONARIO A LOS ESTUDIANTES, A LAS MADRES Y LOS RECORDATORIOS DE ACTIVIDAD FÍSICA DE 24 HORAS PARA ESTUDIANTES de 10 a 14 años de edad en la CIUDAd de MéXıco.*,‡ MéXıCo, 1996}

\begin{tabular}{|c|c|c|c|c|c|c|}
\hline Tipo de actividad & $\begin{array}{l}\text { Media cruda } \\
\text { Recordatorios de } \\
24 \text { horas }\end{array}$ & $\begin{array}{l}\text { M edia cruda } \\
\text { Cuestionario de } \\
\text { los estudiantes }\end{array}$ & $\begin{array}{c}\text { Media cruda } \\
\text { Cuestionario } \\
\text { de las madres }\end{array}$ & $\begin{array}{l}\text { Media ajustada } \\
\text { Recordatorios de } \\
24 \text { horas }\end{array}$ & $\begin{array}{l}\text { M edia ajustada } \\
\text { Cuestionario de } \\
\text { los estudiantes }\end{array}$ & $\begin{array}{l}\text { Media ajustada } \\
\text { Cuestionario de } \\
\text { las madres }\end{array}$ \\
\hline Ver televisión $n^{\S}$ & 2.8 & $4.3^{\#}$ & 3.4 & 3.1 & 4.5 & 3.7 \\
\hline Dormir & 9.1 & 9.0 & 9.5 & 9.2 & 9.3 & 9.6 \\
\hline Leer/hacer tareas & 1.4 & 2.3 & 2.1 & 1.3 & 2.2 & 2.0 \\
\hline Actividad física moderada & 3.2 & 1.2 & 1.2 & 3.2 & 1.2 & 1.2 \\
\hline Caminar & 1.0 & 0.4 & 0.3 & 1.0 & 0.4 & 0.3 \\
\hline Actividad moderada, excluyendo caminar & 2.2 & 0.8 & 1.0 & 2.2 & 0.8 & 1.0 \\
\hline Actividad física vigorosa & 0.5 & 1.0 & 0.7 & 0.5 & 1.0 & 0.8 \\
\hline Total actividad física excluyendo caminar & 2.7 & 1.8 & 1.7 & 2.7 & 1.8 & 1.8 \\
\hline Total actividad física & 3.7 & 2.2 & 2.0 & 3.7 & 2.2 & 2.1 \\
\hline \multicolumn{7}{|c|}{$\begin{array}{l}\text { * Este cuadro incluye datos de } 114 \text { estudiantes que proporcionaron información en al menos una de las siguientes medidas: la segunda aplicación del } \\
\text { cuestionario a los estudiantes, el cuestionario de las madres o los recordatorios de actividad física de } 24 \text { horas } \\
\text { Ajustadas por edad, sexo, ingresos y enfermedad anterior a la aplicación del cuestionario } \\
\$ \text { Incluye ver programas de televisión, pelí́culas o videos en la videograbadora y jugar con videojuegos } \\
\# \text { p } 0.05 \text { para las pruebas de comparación de medias en las respuestas de los cuestionarios de los estudiantes y las madres contra los recordatorios de } 24 \\
\text { horas }\end{array}$} \\
\hline
\end{tabular}


de las madres y el recordatorio de 24 horas fueron ligeramente mayores (cuadro III).

$\mathrm{Al}$ estratificar por edad, sexo e ingresos se encontraron diferencias sólo en las correlaciones entre el cuestionario de los estudiantes y el recordatorio de 24 horas para la actividad física vigorosa, con correlaciones más altas entre las mujeres $(r=0.32$ para las mujeres, $r=0.03$ para los hombres), los estudiantes de primaria ( $r=0.37$ en primaria, $r=0.04$ en secundaria) y de la población de medianos ingresos $(r=0.63$ en la de medianos ingresos, $r=0.04$ en la población de bajos ingresos). El ajuste para el IMC y días hábiles/de descanso no modificó estos resultados.

En total 114 estudiantes respondieron el primer o segundo CAINM; 113 estudiantes completaron la primera aplicación del cuestionario y 105 completaron el segundo seis meses después; de ellos, 104 llenaron el cuestionario en ambas aplicaciones. El análisis de medidas repetidas permitió usar la información de los 114 estudiantes. Las estimaciones del tiempo de ver TV fueron significativamente mayores $(p<0.05)$ y leer 0 hacer tareas fueron menores en el primer cuestionario aplicado, comparado con el segundo. Los coeficientes de reproducibilidad para las distintas formas de actividad e inactividad fueron de 0.09 a 0.55 , y todos resultaron estadísticamente significativos, excepto leer/ hacer tareas (cuadro IV). Tras el ajuste por otras cova- riables, los coeficientes de correlación no se modificaron para otras covariables ni cuando se estratificó por día hábil/día de asueto.

\section{Discusión}

Los resultados de este estudio son consistentes con estudios de validación de otros cuestionarios de actividad física realizados con escolares. En un estudio con niñas de entre 8 y 11 años de edad, las medidas de actividad e inactividad física, obtenidas mediante un cuestionario, tuvieron correlaciones de 0.30 a 0.88 contra diarios de actividad.$^{31}$ La correlación deatenua$\mathrm{da}^{30}$ del tiempo de ver TV, como se informó en un cuestionario y en recordatorios de 24 horas entre estudiantes de cuarto y quinto grado en los Estados Unidos de América (EUA), fue $r=0.54 . *$ Sin embargo, la correlación entre las estimaciones de actividad vigorosa por el CAINM comparadas con las de los recordatorios de 24 horas fue menor que la que se encontró entre el YAQ y tres cuestionarios de 24 horas aplicados a estudiantes de preparatoria en los

* Gortmaker S. Department of Health and Social Behavior, Harvard School of Public Health, Boston (MA), Estados Unidos de América, 1997. Comunicación personal.

\section{Cuadro III

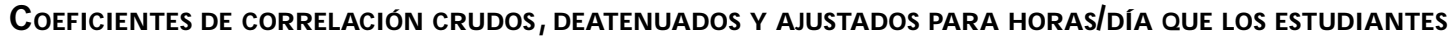 DEDICAN A LOS DISTINTOS TIPOS DE ACTIVIDAD E INACTIVIDAD FÍSICA, COMO SE ESTIMÓ EN LA SEGUNDA APLICACIÓN DEL CUESTIONARIO A LOS ESTUDIANTES, A LAS MADRES Y EN LOS RECORDATORIOS DE ACTIVIDAD FISICA DE 24 HORAS

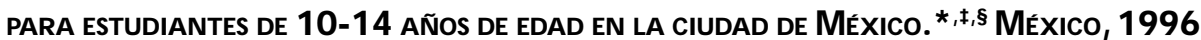

Tipo de actividad

\begin{tabular}{l} 
Cuestionario de los estudiantes- \\
recordatorios de 24 horas \\
\hline Crudos Deatenuados Ajustados
\end{tabular}

Cuestionario de las madresrecordatorios de 24 horas Crudos Deatenuados Ajustados
Cuestionario de los estudiantesruestionario de las madres Crudos Ajustados

\begin{tabular}{|c|c|c|c|c|c|c|c|c|}
\hline Ver televisión\# & $0.55^{\alpha}$ & $0.69 \AA$ & $0.51^{\&}$ & $0.25^{\AA}$ & $0.32^{\&}$ & 0.17 & $0.32^{\AA}$ & $0.26^{\alpha}$ \\
\hline Dormir & 0.19 & 0.25 & 0.14 & 0.13 & 0.17 & 0.10 & $0.43^{\&}$ & $0.39^{\&}$ \\
\hline Leer/hacer tareas & 0.16 & 0.23 & 0.11 & 0.12 & 0.17 & 0.11 & $0.27 \AA$ & $0.26^{\&}$ \\
\hline Actividad física moderada & 0.02 & 0.03 & 0.03 & $0.26^{\S}$ & $0.38^{\&}$ & $0.27^{\S}$ & 0.12 & 0.13 \\
\hline Caminar & 0.04 & 0.08 & 0.04 & 0.22 & $0.42^{\S}$ & 0.24 & $0.22^{\&}$ & $0.22^{\S}$ \\
\hline Actividad moderada excluyendo caminar & 0.05 & 0.11 & 0.06 & 0.22 & 0.49 & 0.21 & 0.12 & 0.14 \\
\hline Actividad física vigorosa & $0.23^{\S}$ & 0.54 & 0.15 & $0.32^{\S}$ & 0.75 & $0.31^{\&}$ & $0.27 \AA$ & 0.17 \\
\hline Actividad moderada y vigorosa excluyendo caminar & 0.04 & 0.07 & 0.04 & 0.18 & 0.30 & 0.18 & 0.09 & 0.06 \\
\hline Actividad moderada y vigorosa & 0.01 & 0.01 & 0.02 & 0.22 & $0.30^{\&}$ & 0.21 & 0.11 & 0.09 \\
\hline
\end{tabular}

* Este cuadro incluye datos de 111 estudiantes que proporcionaron información en al menos dos de las siguientes medidas: la segunda aplicación del cuestionario a los estudiantes, el cuestionario de las madres o los recordatorios de actividad física de 24 horas, tomando en cuenta la existencia de valores perdidos

₹ Las correlaciones deatenuadas son correlaciones crudas deatenuadas por variación intraindividual ${ }^{32}$

$\S$ Las correlaciones ajustadas fueron ajustadas por sexo, grado, ingresos y enfermedad anterior a la aplicación del cuestionario

\# Incluye ver programas de televisión, películas o videos en la videograbadora y jugar con videojuegos

$\& p<0.05$ 


\section{Cuadro IV

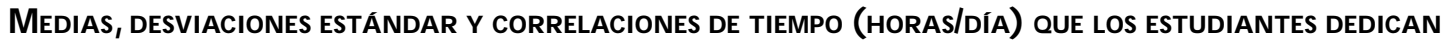 A DIFERENTES TIPOS DE ACTIVIDAD E INACTIVIDAD FÍSICA, COMO SE ESTIMÓ EN LA PRIMERA Y SEGUNDA APLICACIONES del Cuestionario a estudiantes de 10 a 14 años de edad en la ciudad de México.* México, 1996}

\begin{tabular}{|c|c|c|c|c|c|}
\hline \multirow[b]{2}{*}{ Tipo de actividad } & \multicolumn{2}{|c|}{$\begin{array}{c}\text { Primera aplicación } \\
\text { mayo } 1996\end{array}$} & \multicolumn{2}{|c|}{$\begin{array}{l}\text { Segunda aplicación } \\
\text { noviembre } 1996\end{array}$} & \multirow{2}{*}{$\begin{array}{c}\text { Coeficiente de correlación } \\
\text { de Pearson } \\
r\end{array}$} \\
\hline & Media & $\mathrm{DE}$ & Media & DE & \\
\hline Ver televisión ${ }^{\ddagger}$ & 5.1 & 3.4 & $4.3^{\ddagger}$ & 3.5 & $0.53^{\ddagger}$ \\
\hline Dormir & 8.8 & 0.9 & 9.0 & 0.8 & $0.40^{\ddagger}$ \\
\hline Leer/hacer tareas & 1.8 & 1.2 & $2.3^{\ddagger}$ & 1.2 & 0.09 \\
\hline Actividad física moderada & 1.1 & 0.8 & 1.2 & 0.8 & $0.39 \ddagger$ \\
\hline Caminar & 0.3 & 0.3 & 0.4 & 0.4 & $0.43^{\ddagger}$ \\
\hline Actividad moderada excluyendo caminar & 0.8 & 0.7 & 0.8 & 0.6 & $0.38^{\ddagger}$ \\
\hline Actividad física vigorosa & 1.0 & 0.9 & 1.0 & 1.0 & $0.55^{\ddagger}$ \\
\hline Actividad moderada y vigorosa excluyendo caminar & 1.8 & 1.3 & 1.8 & 1.5 & $0.48^{\ddagger}$ \\
\hline Actividad moderada y vigorosa & 2.1 & 1.5 & 2.2 & 1.6 & $0.48^{\ddagger}$ \\
\hline
\end{tabular}

EUA $(r=0.63)^{*}$ o en estudios con adultos. ${ }^{22,23}$ ElCAINM tuvo reproducibilidad aceptable para la mayoría de las formas de inactividad, actividad moderada y vigorosa durante un periodo de seis meses.

Los cuestionarios de actividad física requieren que los estudiantes recuerden sus actividades y que las promedien en relación con un lapso determinado. El cuestionario se centra en formas de actividad en las que puede haber mayor variabilidad entre los escolares. De esta forma, no se evalúa, por ejemplo, el tiempo que pasan los niños sentados en el salón de clase por ser relativamente homogéneo en esta población. Las actividades que son menos comunes o que se distribuyen en cortos periodos durante el día pueden ser más difíciles de recordar y promediar. $^{32} \mathrm{El}$ CAINM mostró una validez pobre para medir actividad física moderada, quizá porque la actividad moderada normalmente se distribuye en periodos cortos durante el día. Por otra parte, se encontraron correlaciones más altas entre el informe del cuestionario y de los recordatorios de 24 horas para actividad vigorosa entre mujeres, estudiantes de primaria y niños de medianos ingresos. Estas diferencias pueden deberse a un mejor informe de actividades en estos grupos, ya sea por el tipo de actividad vigorosa que realizan o estricta-

\footnotetext{
* Field A, Colditz GA, Fox MK, Bosch RJ, Peterson KE. Department of Nutrition, Harvard School of Public Health, Boston (MA), Estados Unidos de América, 1998. Comunicación personal.
}

mente por mayor facilidad para informar su actividad vigorosa en este instrumento.

Este estudio tiene limitaciones que influyen en la interpretación de los resultados. Hubo un mayor índice de participación de la población de bajos ingresos, lo que se asoció con la participación del personal de la escuela en el estudio, y no con otras características de los estudiantes como edad o sexo. No fue posible saber si la participación se asociaba con el IMC.

El estudio reunió información de tres fuentes: los cuestionarios de los estudiantes, los cuestionarios de las madres y los recordatorios de 24 horas. El cuestionario de las madres se refiere a un periodo distinto (julio-agosto de 1996) al de la segunda aplicación del cuestionario de los estudiantes y los recordatorios de actividad de 24 horas (septiembre-noviembre de 1996). El segundo cuestionario de los estudiantes incluye únicamente parte del periodo en el que se reunieron los recordatorios de 24 horas. Estas discrepancias pueden sesgar las estimaciones de asociación hacia el valor nulo; las correlaciones podrían ser mayores si hubieran hecho referencia a los mismos periodos del año.

La validez de una medida puede calcularse al comparar las estimaciones obtenidas por medio de cierto método con las estimaciones medidas con otro. ${ }^{5}$ En el caso de la actividad física se utilizó el recordatorio de actividad física de 24 horas como criterio de comparación, dado que se considera como medida más precisa que los cuestionarios. ${ }^{33}$ Ya que los recordatorios 
de 24 horas dependen de los informes de los estudiantes se introdujo otra medida de comparación y se aplicó un cuestionario a las madres de los estudiantes. Las variables para las que se encontraron correlaciones moderadas o altas entre el cuestionario de los estudiantes y los recordatorios de actividad de 24 horas también mostraron correlaciones altas entre los cuestionarios de los estudiantes y las madres.

Otra limitación del estudio consiste en que la mayoría de los recordatorios con información durante días de asueto fueron reunidos en lunes y, por ello, hacen referencia a los domingos. Sin embargo, el ajuste del tiempo de dormir, leer/hacer tareas y ver TV por efecto del día de la semana no cambió las correlaciones encontradas entre las medidas basadas en distintas fuentes de información. Asimismo, sólo se pudieron llevar a cabo dos recordatorios de 24 horas, los que pueden no ser suficientes para captar la variabilidad intraindividual en la actividad e inactividad física de los estudiantes y, por ello, aumentar el error de medición. Las correlaciones para la actividad física vigorosa aumentaron sustancialmente tras la deatenuación dados los altos índices de variabilidad intra/interindividual, lo que sugiere que el error de medición está atenuando estos resultados.

Los estudiantes que participan en este estudio pudieron experimentar un proceso de aprendizaje, ya que contestaron el cuestionario de actividad/inactividad física dos veces. La repetición de la evaluación de validez comparando los recordatorios de 24 horas con la primera aplicación del CAINM dio resultados similares, aunque las correlaciones fueron menores que las encontradas entre los recordatorios y la segunda aplicación de CAINM.

La evaluación de reproducibilidad se efectuó en un periodo de seis meses en el que es posible que las estudiantes hayan modificado sus patrones de actividad. Los coeficientes de reproducibilidad podrían ser mayores a los encontrados si ésta se hubiese evaluado a un periodo más corto.

Esta investigación encontró que los estudiantes dedican mucho tiempo a ver TV, mientras que realizan durante cortos periodos actividad física moderada y vigorosa. El tiempo promedio de actividad moderada y vigorosa, como se informó en los recordatorios de 24 horas, excede los niveles recomendados para los niños en los EUA (30 minutos/día), ${ }^{34,35}$ aunque se ha documentado una sobrestimación del tiempo de actividad vigorosa en recordatorios de 24 horas. ${ }^{*}$ En el

\footnotetext{
* Gortmaker S. Department of Health and Social Behavior, Harvard School of Public Health, Boston (MA), Estados Unidos de América, 1997. Comunicación personal.
}

cuestionario del estudiante, las estimaciones del tiempo de ver TV, actividad moderada y vigorosa son similares a las que se encontraron entre los estudiantes de EUA. ${ }^{9,36}$

En el cuestionario, los estudiantes sobrestimaron el tiempo de leer, ver TV y de actividad vigorosa, y subestimaron la actividad moderada en comparación con las estimaciones de los recordatorios de 24 horas. El CAINM tuvo una reproducibilidad aceptable para evaluar el tiempo de inactividad, actividad moderada y vigorosa; sin embargo, mostró validez aceptable sólo en la evaluación del tiempo de ver TV. Dados su bajo costo y fácil aplicación, el cuestionario parece ser adecuado para estudios de investigación que pretendan categorizar a los individuos de acuerdo con el tiempo que ven TV dentro de grandes poblaciones de estudiantes en las áreas urbanas de México.

\section{A gradecimientos}

A los estudiantes y personal del Centro de Integración Educativa, Escuela Secundaria Delfina Huerta López, Escuela Primaria sin nombre, clave 41, por la ayuda proporcionada. A Alison Field quien aportó importantes comentarios para el análisis de los datos. A Irma Aldana, Victoria Cervantes, José Luis Xicoténcatl y Edith Yáñez que trabajaron en la recolección de datos y en los procesos de codificación. El registro de datos se realizó en el Instituto Nacional de Salud Pública de México, bajo la supervisión de Antonio García. Noemí Figueroa estuvo a cargo del control de las bases de datos.

\section{Referencias}

1. U.S. Department of Health and Human Services. Physical activity and health:A report of the surgeon general.Atlanta (GA): U.S. D epartment of $H$ ealth and Human Services, Centers for D isease C ontrol and Prevention, $\mathrm{N}$ ational $\mathrm{C}$ enter for $\mathrm{C}$ hronic D isease Prevention and Health Promotion, 1996.

2. Secretaría de Salud. Mortalidad 1996. México D.F.: Secretaría de Salud, 1997.

3. Hernández B, Peterson K, Sobol A, Rivera J, Sepúlveda J, Lezana MA. Sobrepeso en mujeres de 12 a 14 años y niños menores de cinco años en México. Salud Publica Mex 1996;38:178-188.

4. Markovitz JH. Cardiovascular risk factors in rapidly developing countries. Ann Epidemiol 1998:8:1-2.

5. W illet W. N utritional epidemiology. 2a. edición. N ueva York (N Y): 0 Xford University Press, 1998.

6. Gortmaker SL, D ietz W H, C heung L. Inactivity, diet and the fattening of America. J Am D iet Assoc 1990;41:33-35.

7. Blair SN, Kohl HW, Gordon N F. How much physical activity is good for health? Ann Rev Public Health 1992;13:99-126.

8. Dietz W H. Physical activity and childhood obesity. N utrition 1991;7: 295-297.

salud pública de méxico / vol.42, no.4, julio-agosto de 2000 
9. Gortmaker S, Must A, Sobol A, Peterson K, Colditz G, Dietz W. Television viewing as a cause of increasing obesity among children in the $U$ nited States, 1986-1990. Arch Pediatr Adolesc Med 1996;150:356-362.

10. Hernández B, Gortmaker SL, G olditz GA, Peterson KE, Laird N M, Parra-C abrera S.Association of obesity with physical activity, television programs and other forms of video viewing among children in Mexico City. Int J 0 bes 1999;23:845-854.

11. D ietz W H, Gortmaker SL. D o we fatten our children at the television set? 0 besity and television viewing in the children and adolescents. Pediatrics 1985;75:807-812.

12. Sánchez E. Televisión, socialización y educación informal en Guadalajara. En: Rebeil MA, MontoyaA, ed.Televisión y desnacionalización. Colima, México: Universidad de Colima, 1987:101-125.

13. Coleman K, Saelers B,W iedrich-Smith M, Finn J, Epstein LH. Relationships between TricTrac-R3D vectors, heart rate, and self report in obese children. Med Sci Sports Exerc 1997;29:1535-1542.

14. O 'H ara N M, Baranowski T, Simons-Morton BG, W ilson BS, Parcel GS. Validity of the observation of children's physical activity. Res Q Exerc Sport 1989:60:42-51.

15. KriskaAM, C asperson CJ, ed.A collection of physical activity questionnaires for health-related research. Med Sci Sports Exerc 1997;29:S1-S206. 16. Prentice AM, Black AE, C oward HL, D avies GR, G oldberg PR, Murgatroyd J et al. High levels of energy expenditure in obese women. Br Med J 1986;292:983-987.

17. Melanson EL, Freedson PS. Physical activity assessment: A review of methods. Crit Rev Food Sci N utr 1996;36:385-396.

18. Gortmaker S, Peterson K, W iecha J, Sobol AM, Dixit S, Fox MK et al. Reducing obesity via a school-based interdisciplinary intervention among youth: Planet Health. Arch Pediatr A dolesc Med 1999;153(4):409-418. 19. Habicht JP. Estandarización de métodos epidemiológicos cuantitativos sobre el terreno. Bol 0 ficina Sanit Panam 1974;76:375-385.

20. Lohman TG, Roche AF, Martorell R, ed. Anthropometric Stantardization Reference Manual. Champaign (IL): Human Kinetics Books, 1988.

21. Chasen-Taber S, Rimm E, Stampfer J et al. Reproducibility and validity of a self-administered physical activity questionnaire for male health professionals. Epidemiology 1996;7:81-86.

22. W olf AM, Hunter CJ, Colditz GA et al. Reproducibility and validity of a self-administered physical activity questionnaire. Int I Epidemiol 1994; 23:991-999.

23. Ainsworth $B E$, Haskell W L, Leon AS, et. al. Compendium of physical activities. Med Sci Sports Exerc 1993;25:71-80.
24. Must A, D allal GE, D ietz W H. Reference data for obesity: $85^{\text {th }}$ and $95^{\text {th }}$ percentiles of body mass index (wth/ht ${ }^{2}$ ) -and triceps skinfold thickness. Am J Clin N utr 1991;53:839-846.

25. Must A, D allal GE, D ietz W H. Reference data for obesity: $85^{\text {th }}$ and $95^{\text {th }}$ percentiles of body mass index (wth/ht $\left.{ }^{2}\right)$-a correction. Am J Clin N utr 1991:54:773.

26. SAS Institute. Statistical Anaylisis Software: Mixed models reference Manual. Cary N C: SA S Institute, 1996.

27. Fitzmaurice $G M$, Laird N M, Z ahner GE, D askalakis C. Bivariate logistic regression analysis ratings using multiple informants. Am J Epidemiol 1995;142:1194-1203.

28. C naan A, Laird N M, Slasor P. U sing the general linear mixed model to analyse unbalanced repeated measures and longitudinal data. Stat Med 1997;16:2349-2380.

29. Beaton GH, Milner J, Corey P, MCGuire V, Cousins M, Stewart E et al. Sources of variance in 24-hour dietary recall data: Implications for nutrition study design and interpretation. Am J C lin N utr 1979;32:2456-2559. 30. Rosner B,W illett W C. Interval estimates for correlation coefficients corrected for within-person variation: Implications for study design and hypothesis testing. Am J Epidemiol 1988;127:377-386.

31. Ching PL. Reliability and relative validity assessment of physical activity measures in pre-adolescent girls (tesis doctoral). Boston (MA): Escuela de Salud Pública de Harvard, 1992.

32. Peterson KE, Field AE, Fox KM, Black B, Simon DS, Bosch RB et al. Validation of YRBSS questions on dietary behaviors and physical activity among adolescents in grades 9-12. Boston (MA): Escuela de Salud Pública de Harvard. Prepared for Centers for Disease Control and Prevention, Division of Adolescent and School Health, 1996.

33. W eston A, Petosa R, Pate R.Validation of an instrument for measurement of physical activity in youth. Med Sci Sports Exerc 1997;29:138-143. 34. Kolbe LJ, Kann L, Collins JL. O verview of the Youth Risk Behavior Surveillance System. Public Health Rep 1993;108(Suppl 1):2-10.

35. Kolbe LJ.An epidemiological surveillance system to monitor the prevalence of youth behaviors that most affect health. Health Educ 1990;21: 44-48.

36. Shannon B, Peacock J, Brown MJ. Body fatness, television viewing and calorie-intake of sample of Pennsylvania sixth grade children. J N utr Educ 1991;23:262-268. 